\title{
Singlet Excitation Transfer in Columnar Liquid Crystals Studied by Monte Carlo Simulations
}

\author{
Hervé Sigal and Dimitra Markovitsi* \\ CEA Saclay, DRECAM, SCM, CNRS-URA 331, Laboratoire de Photophysique et Photochimie, \\ 91191 Gif-sur-Yvette, France
}

Lazaros K. Gallos and Panos Argyrakis

Department of Physics, University of Thessaloniki, 54006 Thessaloniki, Greece

Received: December 25, 1995; In Final Form: March 28, $1996^{\otimes}$

The present paper deals with electronic excitation transfer in columnar liquid crystals formed by disklike molecules. The transport process is considered to occur in the singlet state via random walk hopping and is studied by Monte Carlo simulations. The distance dependence of the hopping probability is determined by the extended dipole approximation. Long-range steps, both intracolumnar and intercolumnar, are taken into account. The influence of (i) the number of nearest neighbors to which hops may occur, (ii) the intercolumnar distance, (iii) the length and the orientation of the transition dipoles, on the root mean square displacement along the column axis and the survival probability in presence of traps is investigated. It is shown that long-range hops slow down the transfer process. The transport is initially one-dimensional and becomes three-dimensional at longer times. The crossover regime is shifted to shorter times when the intercolumnar distance decreases or the length of the transition dipoles increases. The motion of the excitation is accelerated either by a better ordering of the transition dipoles around the column axis or by a continuous change of their orientation during the walk.

\section{Introduction}

A large number of publications deal with energy transport in restricted geometries. Among the various systems studied experimentally in this respect, columnar liquid crystals have attracted attention because of their highly anisotropic structure ${ }^{1}$ (Figure 1). Indeed, these molecular materials have been considered as systems in which excitation transport should be one-dimensional. $^{2-8}$ Such an assumption seems to be quite reasonable for triplet migration occurring via the short-range exchange interactions. ${ }^{7}$ Conversely, for singlet states, the ideal picture of a one-dimensional process turned out to be oversimplified. ${ }^{9}$

The most detailed investigation of singlet excitation transport in columnar liquid crystals was carried out for hexakis(alkoxy)triphenylenes for which energy migration takes place via random walk hopping. ${ }^{8,9}$ The fluorescence decays of these mesophases containing energy traps were fitted by theoretical curves, the fitting parameter being the hopping time between two neighboring molecules in the same column. At a first approximation, a one-dimensional random walk model allowing hops only to first nearest neighbors was tested. ${ }^{8}$ This model gave quite good fits, but the resulting hopping time (30 fs) was too small for an incoherent process. ${ }^{10}$ Following this intriguing result and in order to take into account the different factors playing a role in the excitation transport, Monte Carlo simulations were used to fit experimental fluorescence decays. ${ }^{9}$ Long-distance hops, both intracolumnar and intercolumnar, were considered. Moreover, various patterns were used for the distance dependence of the hopping probability, all corresponding to Coulombic interactions and all giving good fits. The type of those patterns proved to be quite crucial for the hopping time values which were found to vary from several femtoseconds to picoseconds.

\footnotetext{
${ }^{\otimes}$ Abstract published in Advance ACS Abstracts, June 1, 1996.
}

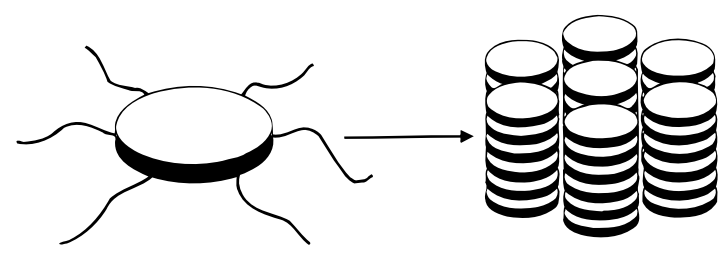

Figure 1. Columnar liquid crystals are usually composed of disklike molecules containing a flat and rigid core, surrounded by flexible chains. Their structure corresponds to stacks of segregated columns separated by the chains in a liquid state. The intercolumnar distance is $15-40$ $\AA$, depending on the lateral chain length, while the stacking distance is smaller than $4.5 \AA$. Columnar mesophases are stable at a certain temperature range; upon cooling, crystallization takes place, resulting in a change of the molecular arrangement. In some cases, the columnar structure can be frozen (glassy state).

The most important conclusion of the above mentioned analysis of experimental data was that molecules cannot be considered as simple points in the description of the transfer process. ${ }^{9}$ Indeed, in columnar phases, the stacking distance is smaller than the "diameter" of the disklike chromophores. Therefore, the orientation and the length of transition dipoles have to be taken into account. The effect of those two factors on the properties of the eigenstates of columnar aggregates formed by triaryl pryrylium salts has been already depicted in ref 11 in which the extended dipole approximation ${ }^{12}$ was used.

Within this context, we decided to use Monte Carlo simulations to study in detail the singlet transport occurring in columnar phases via a hopping mechanism. We determine the hopping probability by the extended dipole approximation, and we neglect exchange interactions. We mainly focus on two relevant quantities, the root mean square displacement along the column axis $\left(R_{z}\right)$ and the survival probability of the excitation in presence of traps $(\Phi) .{ }^{13,14}$ The former quantity allows us to illustrate the temporal and spatial evolution of the excitation within those molecular materials, while the latter is related to measured 


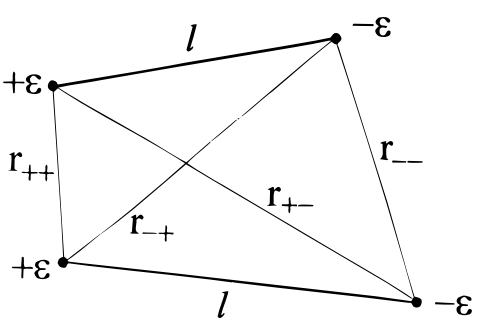

Figure 2. Schematic representation of the extended dipole model. The transition moment is approximated by a dipole composed of two charges $+\epsilon$ and $-\epsilon$ separated by a distance $l$ and placed at the center of the molecule. The coupling $V$ between the two transition moments is given by the electrostatic interaction between the four charges (eq 2).

fluorescence decays. The parameters whose influence on $R_{z}$ and $\Phi$ is studied are (i) the number of nearest neighbors $(n)$ to which hops may occur, (ii) the intercolumnar distance, and (iii) the length and the orientation of the transition dipoles around the column axis. We also quantify the one-dimensional character of the transfer process by calculating the probability of the excitation to remain in the same column $\left(P_{1 \mathrm{~d}}\right)$ as a function of time.

Various types of columnar liquid crystals exist. ${ }^{1}$ In the present investigation, we are interested in $D_{\text {ho }}$ mesophases, illustrated on Figure 1, because energy transport has been studied experimentally in such systems. ${ }^{8,9}$ In $\mathrm{D}_{\text {ho }}$ mesophases, the stacking distance is constant, the aromatic cores are centered and perpendicular to the column axis, and the columns are arranged in a hexagonal array.

The paper is organized as follows. In section 2, we determine the distance dependence of the hopping probability in the extended dipole approximation and we present the various patterns related to the orientation of the transition dipoles. The procedure followed in the Monte Carlo simulations is described in section 3. In section 4, we report and discuss the results of the calculations. In section 5, we summarize our approach and we draw the final conclusions.

\section{Hopping Probability}

Energy transfer takes place through a hopping mechanism when the coupling $V$ between transition moments is weaker than the Boltzmann factor. If the transfer is slow compared to vibrational relaxation, the hopping rate $k_{\mathrm{h}}$ is given by the Fermi golden rule:

$$
k_{\mathrm{h}}=4 \pi^{2} \rho V^{2} / h
$$

where $\rho$ is the density of final states. The hopping time $t_{\mathrm{h}}$ is the reciprocal of the hopping rate $\left(t_{\mathrm{h}}=1 / k_{\mathrm{h}}\right)$ and the hopping probability is proportional to the hopping rate. Equation 1 gives the possibility of correlating the hopping probability to the coupling and, consequently, to transition moments.

In the extended dipole model, ${ }^{12}$ a transition moment is approximated by a dipole composed of two charges $+\epsilon$ and $-\epsilon$ separated by a distance $l$ and placed at the center of the molecule. The dipole moment $\mu$ is equal to $\epsilon l$, and the coupling $V$ between two transition moments is given by the electrostatic interaction between the corresponding four charges (Figure 2):

$$
V=K\left(\frac{1}{r_{++}}+\frac{1}{r_{--}}-\frac{1}{r_{+-}}-\frac{1}{r_{-+}}\right)
$$

where $K$ is a constant. It should be stressed that the dipole length used in the extended dipole approximation is not the length of the transition dipole in a quantum mechanical sense. It corresponds to an effective length providing a distance

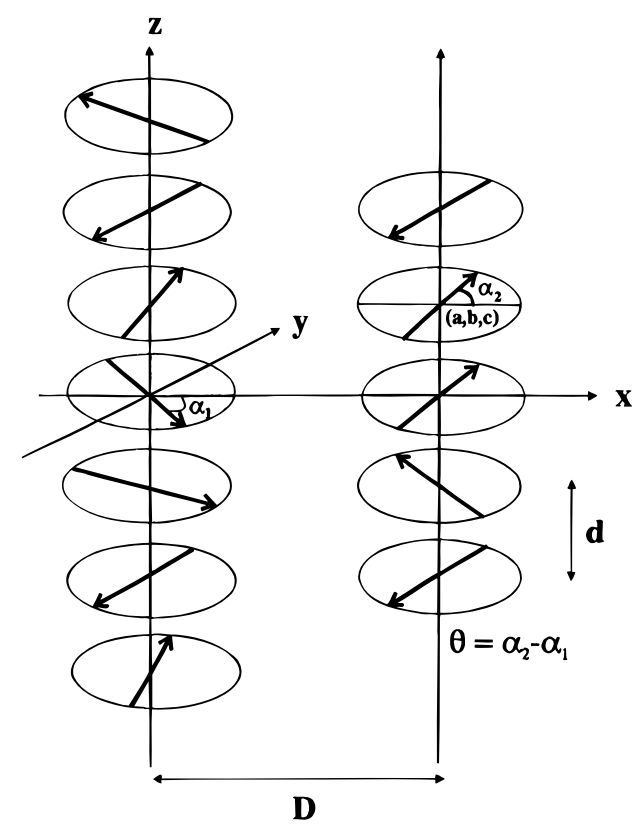

Figure 3. Schematic representation of the molecules considered in the calculation of the hopping probability for $n=3$. Excitation is initially located at the origin of a Cartesian coordinate system (disk in grey) and can jump to any of the represented molecules in the same or in one of the six neighboring columns. For simplicity, only one of the six neighboring columns is shown. Arrows denote the transition dipoles. The angle $\theta$ used in eqs $3-6$ is defined as $\theta=\alpha_{2}-\alpha_{1}$. The position to which excitation hops has coordinates $(a, b, c)$.

dependence of the coupling equivalent to the quantum mechanical one which contains not only dipole-dipole but also higher terms (dipole-quadrupole, quadrupole-quadrupole, octupoleoctupole, etc.). ${ }^{9}$

The transition dipoles of two molecules belonging to a $D_{\text {ho }}$ phase are parallel to the $x y$ plane (Figure 3 ). If they are located at positions $(0,0,0)$ and $(a, b, c)$ of a Cartesian coordinate system, the distances $r_{++}, r_{--}, r_{+-}$, and $r_{-+}$, appearing in eq 2 , can be written as follows:

$$
\begin{aligned}
& r_{++}=\left[\left(a-\frac{l}{2}(\cos \theta-1)\right)^{2}+\left(b-\frac{l}{2} \sin \theta\right)^{2}+c^{2}\right]^{1 / 2} \\
& r_{--}=\left[\left(a+\frac{l}{2}(\cos \theta-1)\right)^{2}+\left(b+\frac{l}{2} \sin \theta\right)^{2}+c^{2}\right]^{1 / 2} \\
& r_{+-}=\left[\left(a-\frac{l}{2}(\cos \theta+1)\right)^{2}+\left(b+\frac{l}{2} \sin \theta\right)^{2}+c^{2}\right]^{1 / 2} \\
& r_{-+}=\left[\left(a+\frac{l}{2}(\cos \theta+1)\right)^{2}+\left(b-\frac{l}{2} \sin \theta\right)^{2}+c^{2}\right]^{1 / 2}
\end{aligned}
$$

The angle $\theta$ is the difference $\theta=\alpha_{2}-\alpha_{1}$; the angles $\alpha_{1}$ and $\alpha_{2}$ are defined in Figure 3. Equations 1-6 allow us to determine the probability of the excitation to hop from one molecule to another as a function of their position and their orientation within the columnar lattice.

Regarding the orientation of transition dipoles around the column axes, we examine three different patterns, related to the molecular orientation in columnar liquid crystals: "random and frozen", "randomizing", and "helical".

In the first one, the transition dipoles are randomly distributed around the column axis and they do not move during the transfer process. Experimentally, such a pattern typically corresponds to a glassy state, in which molecular movements are hindered. ${ }^{15}$

In the second pattern, the transition dipoles are randomly distributed and they reorientate after each hop of the excitation. 
Randomizing structures have been used in the study of random walks in polymeric systems. ${ }^{16,17}$ This pattern is appropriate for liquid crystalline columnar phases, in which various types of molecular motions occur during the hopping process. ${ }^{18,19}$

Finally, in the helical pattern, the angle between two neighboring transition dipoles is constant and equal to $45^{\circ}$. Such helical structures have been determined by X-ray diffraction measurements for certain columnar phases formed by triphenylene derivatives. ${ }^{20,21}$

\section{Simulation Procedure}

A "particle" corresponding to the excitation, localized on a single molecule, is considered to perform a random walk on a three-dimensional lattice consisting of $10^{6}$ sites (Figure 3 ). The sites are aligned along the $z$ direction at a distance of $3.6 \AA$. The column axes form a hexagonal array in the $x y$ plane. The intercolumnar distance is varied between 15 and $35 \AA$. We use cyclic boundary conditions in the $x$ and $y$ directions and reflecting ones in the $z$ direction.

The "particle" is given the possibility of jumping to $2 n$ sites in the same column ( $n$ above and $n$ below the current position) and to $2 n-1$ sites of the six neighboring columns (Figure 3). The number of nearest neighbors $n$ is varied between 1 and 12 . The distance dependence of the hopping probability is determined as explained in section 2 . The lengths of the extended dipole tested are $1,2,3,4,5$, and $6 \AA$. The point dipole $(l=$ $0)$ is simulated in the calculations by setting $l=10^{-3} \AA$.

For the calculation of the survival probability, we consider that $1 \%$ of the lattice sites are energy traps acting with the trapping efficiency equal to 1 . Traps are randomly distributed in the lattice. We monitor the time needed for a particle to be trapped. The same procedure is repeated $10^{5}-10^{6}$ times, for different trap distributions. Then, we calculate the percentage of the particles which have not been trapped at time $t$, as a function of time. In the plots presented below, we do not take into account the lifetime of the excitation in the absence of traps (intrinsic lifetime). The estimated error on the data is of the order of $1 \%$ for the root mean square displacement along the column axis $\left(R_{z}\right)$ and $0.5 \%$ for the survival probability $\Phi$.

In order to make the connection with the real systems clear, we use physical units for the distance $(\AA)$ and time (ns). For this purpose, we have assumed that the hopping time between two nearest neighbors in the stack corresponding to parallel transition dipoles is 1 ps. ${ }^{9}$ We present the results of our calculations on a time scale of $0.1-100 \mathrm{~ns}$, corresponding to usual experimental conditions for recording fluorescence decays.

\section{Results and Discussion}

4.1. Number of Neighbors. At first, we examine the role played by the number of nearest neighbors $n$ to which excitation can hop. This question has been addressed previously but in relation to different basic quantities of the random walk than those presented here. ${ }^{22,23}$ Figure 4 shows the influence of $n$ on the root mean square displacement and the survival probability. We can see that when $n$ increases, $R_{z}$ becomes smaller, while $\Phi$ increases. In other terms, the higher the $n$ used in the simulations, the more restricted appears the motion of the excitation. This happens because the time spent for a longrange hop, for example, to the 10th neighbor, is considerably longer than the time needed by the particle to cover the distance of 10 lattice sites with steps to the first neighbor only.

We observe that, at a given time, the curves in Figure 4 converge for a certain value of $n$; for example, at $100 \mathrm{~ns}$, convergence occurs for $n=10$. Thus, including more than 10 neighbors in the calculations does not affect the profile of $R_{z}$
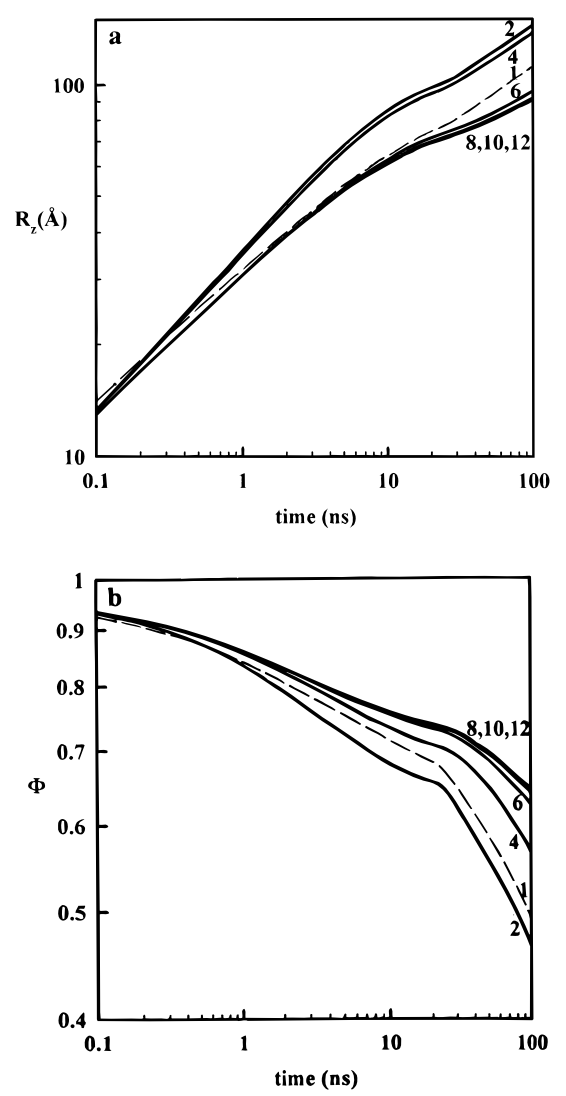

Figure 4. Influence of the number of nearest neighbors $(n=1,2,4$, $6,8,10,12$ ) to which excitation may jump on (a) the root mean square displacement along the column axis $\left(R_{z}\right)$ and (b) the survival probability $\Phi$ for a trap concentration of $10^{-2}$. The dashed line corresponds to the first nearest neighbor approximation. Conditions: "random and frozen" orientation of transition dipoles, $l=3 \AA, D=25 \AA$.

and $\Phi$ at the time scale in which we are interested. Indeed, the probability of hopping to further neighbors $(n>10)$ is so small that it is never realized within the examined time scale.

It is worth noticing that the curves obtained for $n=1$ do not follow the monotonicity of $n$. This happens because there is a relatively high probability of finding two successive molecules in the same column whose transition dipoles form an angle close to $90^{\circ}$, somewhere above the current position and somewhere below it. The hopping probability is then too small, and these sites act as infinitely high barriers. The particle is thus confined in the region between these two barriers, and its motion is limited. When $n \geq 2$, the particle is not hampered by the presence of these high barriers, since it may easily jump over.

Finally, we observe in Figure 4 that the plots of both $R_{z}$ and $\Phi$ present crossovers around $10 \mathrm{~ns}$. This behavior will be discussed in the following paragraph (section 4.2), together with the results shown in Figure 5.

4.2. Intercolumnar Distance. Figure 5 shows the influence of intercolumnar distance $D$ on $R_{z}$ and $\Phi$. For comparison reasons, the behavior of a one-dimensional system $(D=\infty)$ characterized by a dipole length of $3 \AA$ and "random and frozen" orientation of transition dipoles is also illustrated. In the latter case, a straight line is observed and $R_{z}$ varies as $t^{0.4}$. Such a variation is different from that expected $\left(R_{z} \propto t^{0.5}\right)$ for a random walk on a one-dimensional system with steps only to first nearest neighbors without taking into account the size and the orientation of transition dipoles. ${ }^{24}$ It is clear that those factors make the transfer process slower.

At early times, the curves plotted in Figure 5a for different $D$ values coincide with the straight line corresponding to the one-dimensional behavior. As time passes, they deviate from 

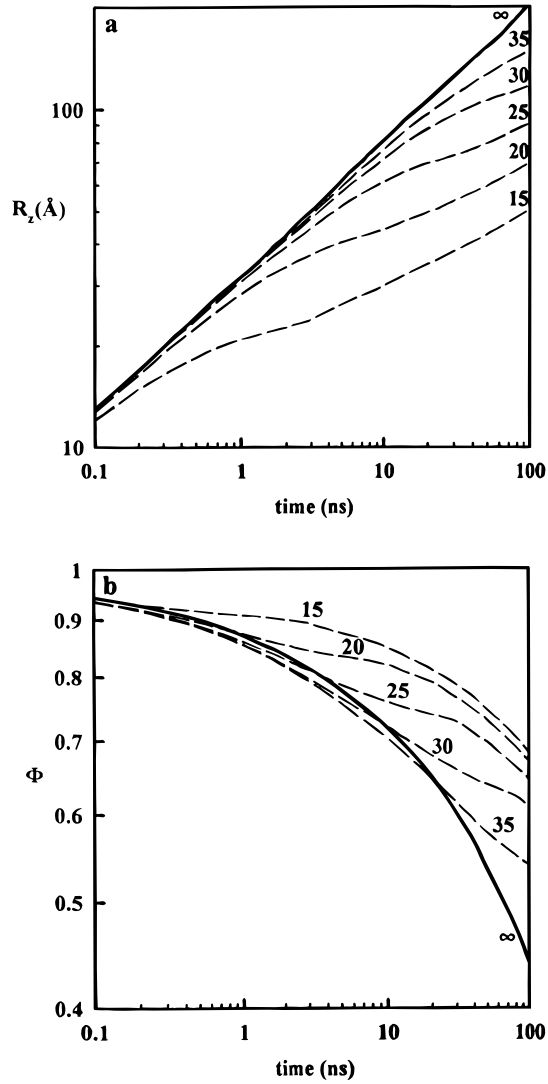

Figure 5. Influence of the intercolumnar distance $(D=15,20,25$, $30,35 \AA$ ) on (a) the root mean square displacement along the column axis $\left(R_{z}\right)$ and (b) the survival probability $\Phi$ for a trap concentration of $10^{-2}$. The solid line corresponds to a one-dimensional system $(D=$ $\infty)$. Conditions: "random and frozen" orientation of transition dipoles, $l=3 \AA, n=10$.

it, because excitations start departing from the column where they were initially formed. When $D$ decreases, the deviation from the one-dimensional behavior is observed at earlier times. In fact, upon decreasing $D$, the probability of intercolumnar jumps increases and the transfer process becomes threedimensional earlier. Crossover regimes are reported in the literature for other types of systems. ${ }^{25,26}$ When intercolumnar jumps become effective, the transfer rate along the column axis, $v_{z}$, defined as

$$
v_{z}=\partial R_{z} / \partial t
$$

diminishes.

We observe in Figure 5b that the decay becomes slower when $D$ decreases. As a matter of fact, the smaller the intercolumnar distance, the more rapid the transition toward the three-dimensional behavior; such a behavior involves long-lasting intercolumnar hops which contribute to the elongation of the particle lifetime. Therefore, at a given time, the closer the columns, the higher the $\Phi$ value. We also remark that all of the decay curves are quite different from that corresponding to the one-dimensional system $(D=\infty)$, even at short times.

In order to obtain a better picture about the dimensionality of the examined system, we have calculated the probability $\left(P_{1 \mathrm{~d}}\right)$ for the excitation to remain in the column in which it has been created as a function of time (Figure 6). We observe that only for $D>30 \AA$, the transport is quasi-one-dimensional $\left(P_{1 \mathrm{~d}}>\right.$ $0.9)$ during the first few nanoseconds. For distances $20 \AA<D$ $<25 \AA$, corresponding to the triphenylene columnar phases studied previously, $2,950 \%$ of the excitations have performed

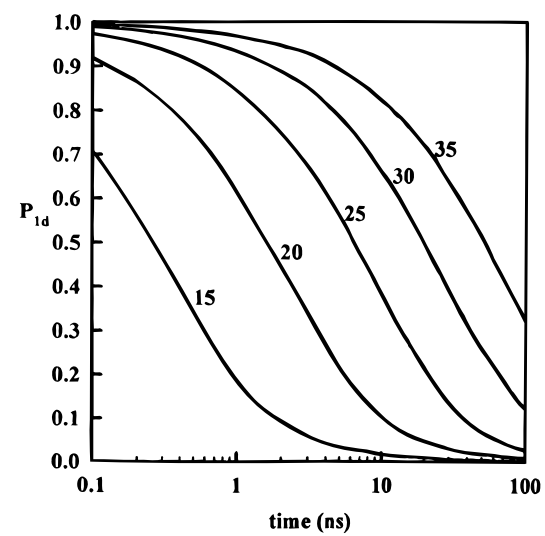

Figure 6. Influence of the intercolumnar distance $(D=15,20,25$, $30,35 \AA$ ) on the probability for the excitation to remain in the same column $\left(P_{1 \mathrm{~d}}\right)$ as a function time. Conditions: "random and frozen" orientation of transition dipoles, $l=3 \AA, n=10$.
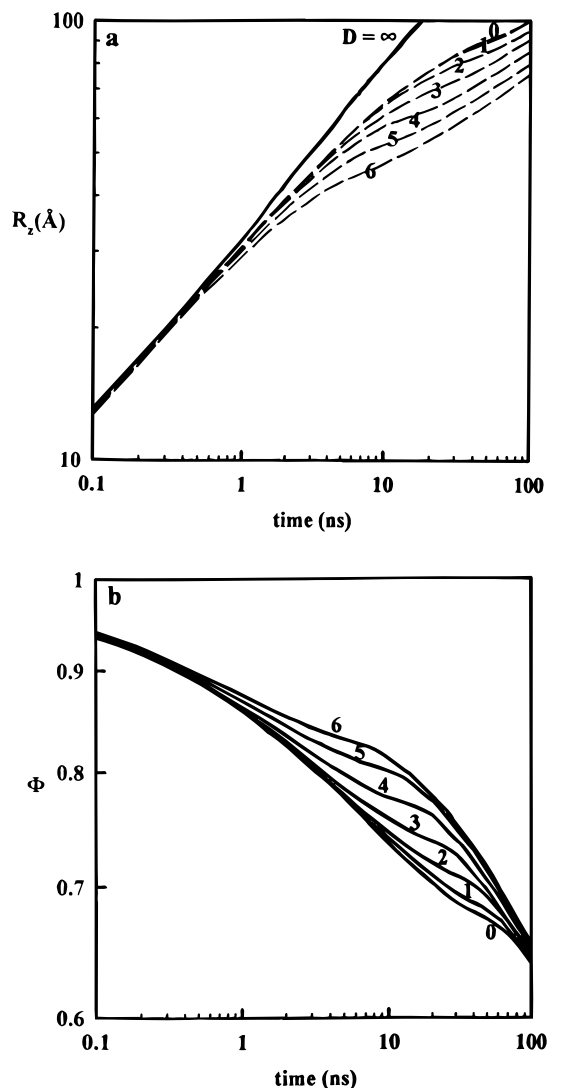

Figure 7. Influence of the length of the extended dipole $(l=0,1,2$, $3,4,5,6 \AA$ ) on (a) the root mean square displacement along the column axis $\left(R_{z}\right)$ and (b) the survival probability $\Phi$ for a trap concentration of $10^{-2}$. Conditions: "random and frozen" orientation of transition dipoles, $D=25 \AA$, $n=10$. The solid line $(D=\infty)$ in (a) corresponds to a one-dimensional system characterized by random and frozen orientation of transition dipoles and $l=3 \AA$ and $n=10$.

intercolumnar jumps within the first few nanoseconds. This confirms that the one-dimensional model is inappropriate for fitting fluorescence decays of these systems.

4.3. Length and Orientation of Transition Dipoles. Figure 7 shows the influence of the extended dipole length on $R_{z}$ and $\Phi$. We remark that, in the one-dimensional region, the transfer rate $v_{z}$ does not depend on the dipole length (Figure 7a). Conversely, $l$ has an influence on the time at which the crossover toward the three-dimensional behavior takes place: the longer the $l$, the earlier the intercolumnar jumps become effective. At longer times, when the three-dimensional regime is developed, 

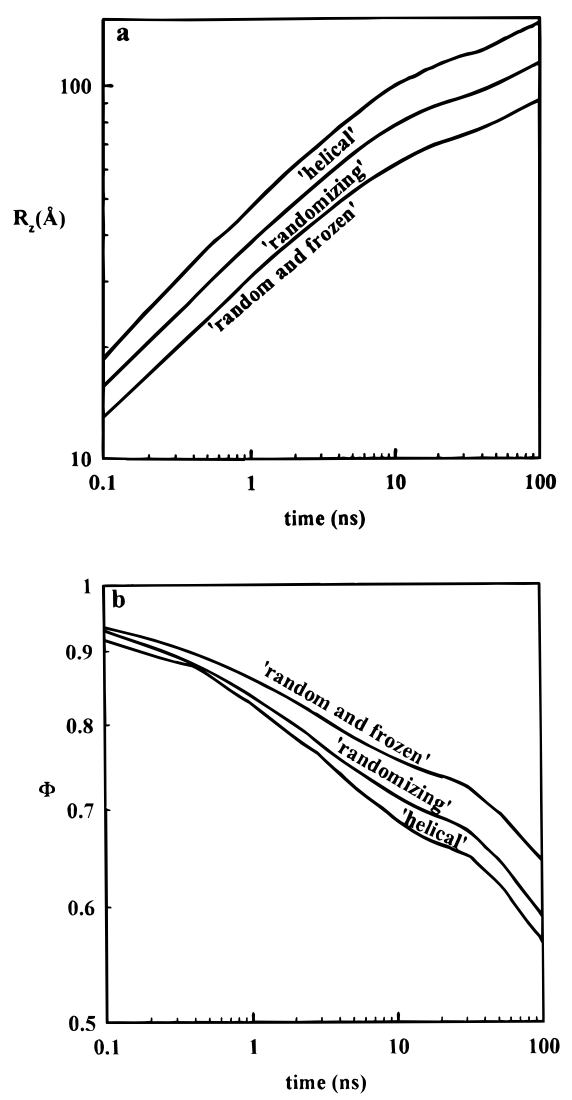

Figure 8. Influence of the orientation of the transition dipoles on (a) the root mean square displacement along the column axis $\left(R_{z}\right)$ and (b) the survival probability $\Phi$ for a trap concentration of $10^{-2}$. Conditions: $l=3 \AA, D=25 \AA, n=10$; "random and frozen", the transition dipoles are randomly distributed around the column axis and they do not move during the transfer process; "randomizing", the transition dipoles are randomly distributed and they reorientate after each hop of the excitation; "helical", the angle between two neighboring molecules in the same column is $+45^{\circ}$.

$v_{z}$ becomes again independent of $l$. The survival probability curves (Figure 7b) coincide at early times; then they split up, and they meet again at longer times. This indicates that $l$ does not affect $\Phi$, neither during the one-dimensional behavior nor the three-dimensional one, but it does play an important role during the crossover regime. At a given time during this regime, $\Phi$ increases for increasing $l$. Thus, regarding the time at which the crossover regime appears, increasing $l$ has the same effect on $R_{z}$ and $\Phi$ as that of decreasing $D$. This suggests that when the dipole length increases, the average interaction between transition moments of chromophores located in neighboring columns becomes stronger and, consequently, the probability of intercolumnar jumps becomes higher.

In Figure 8 we examine three different patterns describing the orientation of the transition dipoles (cf. section 2). For all of the plots presented previously, the frozen and random pattern has been used. When the transition dipoles remain frozen during the transport, but instead of being randomly distributed around the column axis, they form a helix in which the angle between two neighboring dipoles is $45^{\circ}, R_{z}$ increases while $\Phi$ decreases. This means that an increase in the order inside the column will increase the distance to which energy can migrate and will decrease the survival probability because the barriers, generated by unfavorable orientation of transition dipoles, disappear. The same trend is observed if the transition dipoles remain randomly distributed but they reorientate after each hop. The difference in the behavior between a random and frozen and a randomizing pattern is explained as follows. In the former, when the particle finds itself in an environment unfavorable for its motion, it spends a long time in it, while in the latter, the local environment changes continuously and it is easier for the particle to escape.

Finally, we can make a general remark concerning the absolute values related to the root mean square displacement along the column axis and the survival probability in the presence of traps (Figures 5, 7, and 8).

We can see that $R_{z}$ does not exceed $200 \AA$ (56 molecules) in the examined time scale. Such small $R_{z}$ values show that, for nonaligned samples, the motion of the excitation will be practically restricted within a single monodomain whose size is of the order of the micrometers. This is in agreement with fluorescence anisotropy measurements ${ }^{9}$ and also explains why macroscopic alignement of the columnar phases containing traps does not affect the fluorescence decays. ${ }^{25}$

The survival probability at $100 \mathrm{~ns}$ is still high $(>0.5)$. Consequently, we deduce that fluorescence of columnar phases containing $10^{-2}$ traps decays mainly because of the intrinsic lifetime of the fluorescent state- usually a few nanoseconds-and not because of the trapping process.

\section{Summary and Conclusions}

In the present work we have investigated singlet excitation transport in columnar phases using Monte Carlo simulations. This computational technique has enabled us to take into account the complex structure of the examined systems, to limit the number of approximations and, therefore, to construct a model close to reality. We have assumed that the transfer process takes place via random walk hopping, and we have taken into account long-distance hops, both intracolumnar and intercolumnar. Considering that the stacking distance is smaller than the "diameter" of the disklike molecules, we have determined the distance dependence of the hopping probability in the extended dipole approximation.

Our study has been focused mainly on two properties of the random walk, the root mean square displacement along the column axis and the survival probability in the presence of traps. We have presented our results using physical units for the distance $(\AA)$ and time (ns) so that the connection with experimental systems is as clear as possible.

We have shown that long-range interactions are quite important and can change significantly the results of simulations. In general, the transfer process appears to be more rapid if we neglect long-distance hops. Therefore, results based on first nearest neighbor approaches should be considered with care.

We have drawn the conclusion that the system can be treated as one-dimensional only for short times, of the order of a nanosecond or less. Energy transport seems to take place initially inside one column. Afterward, we observe a transition regime where excitations start jumping to neighboring columns, and finally excitations move in the three-dimensional space. This behavior is influenced by both the intercolumnar distance $D$ and the length $l$ of the transition dipole: the three-dimensional character of the walk is delayed in time by increasing $D$ or by decreasing $l$. Finally, we have evidenced that either a better ordering of the transition dipoles or a continuous change in their orientation makes the transport process more rapid.

Acknowledgment. This work has been performed in the frame of a European COST D4 project (design and preparation of new molecular systems with unconventional electrical, optical, and magnetic properties) entitled "Columnar Liquid Crystals as Energy Guides for Molecular Electronics". The authors thank Dr. P. Millié for helpful discussions. 


\section{References and Notes}

(1) Chandrasekhar, S.; Ranganath, G. S. Rep. Prog. Phys. 1990, 53, 57.

(2) Markovitsi, D.; Rigaut, F.; Mouallem, M.; Malthête, J. Chem. Phys. Lett. 1987, 135, 236.

(3) Blanzat, B.; Barthou, C.; Tercier, N.; André, J. J.; Simon, J. J. Am. Chem. Soc. 1987, 109, 6193.

(4) Markovitsi, D.; Lécuyer, I.; Clergeot, B.; Jallabert, C.; Strzelecka, H.; Veber, M. Liq. Cryst. 1989, 6, 83.

(5) Blasse, G.; Dirksen, G. J.; Meijerink, A.; Van der Pol, J. F.; Neelman, E.; Drenth, W. Chem. Phys. Lett. 1989, 154, 420.

(6) Gregg, B. A.; Fox, M. A.; Bard, A. J. J. Phys. Chem. 1989, 93, 4227.

(7) Markovitsi, D.; Lécuyer, I.; Simon, J. J. Phys. Chem. 1991, 95, 3620.

(8) Markovitsi, D.; Lécuyer, I.; Lianos, P.; Malthête, J. J. Chem. Soc., Faraday Trans. 1991, 87, 1785.

(9) Markovitsi, D.; Germain, A.; Millié, P.; Lécuyer, P.; Gallos, L. K.; Argyrakis, P.; Bengs, H.; Ringsdorf, H. J. Phys. Chem. 1995, 99, 1005. (10) Förster, T. In Comparative Effects of Radiation; Burton, M., KirbySmith, J. S., Magee, J. L., Eds.; John Wiley and Sons, Inc.: New York, 1960; pp 300-319.

(11) Ecoffet, C.; Markovitsi, D.; Millié, P.; Lemaistre, J. P. Chem. Phys. 1993, 177, 629 .

(12) Czikklely, V.; Försterling, H. D.; Kuhn, H. Chem. Phys. Lett. 1970, 6,207
(13) Weiss, G. H.; Rubin, R. J. In Advances in Chemicals Physics; Prigogine, I., Rice, S. A., Eds.; John Wiley and Sons, Inc.: New York, 1983; Vol. 52, pp 363-505.

(14) Blumen, A.; Klafter, J.; Zumoffen, G. In Optical Spectroscopy of Glasses; Zschokke, I., Ed.; D. Reidel: Dordecht, The Netherlands, 1986; pp 199-265.

(15) Leisen, J.; Werth, M.; Boeffel, C.; Spiess, H. W. J. Chem. Phys. 1992, 97, 3749 .

(16) Baumann, J.; Fayer, M. D. J. Chem. Phys. 1986, 85, 4087.

(17) Nitzan, A.; Druger, S. D.; Ratner, M. A. Philos. Mag. B 1987, 56, 853.

(18) Richards, G. M.; Dong, R. Y. Liq. Cryst. 1989, 5, 1011.

(19) Dong, R. Y.; Goldfarb, D.; Moseley, M. E.; Luz, Z.; Zimmermann, H. J. Phys. Chem. 1984, 88, 3148.

(20) Levelut, A. M. J. Chim. Phys. 1983, 80, 149.

(21) Heiney, P. A.; Fontes, E.; De Jeu, W. H.; Riera, A.; Carroll, P.; Smith, A. B., III. J. Phys. (Paris) 1989, 50, 461.

(22) Blumen, A.; Zumofen, G. J. Chem. Phys. 1981, 75, 892.

(23) Zumofen, G.; Blumen, A. J. Chem. Phys. 1982, 76, 3713

(24) Chandrasekhar, S. Rev. Mod. Phys. 1943, 15, 1.

(25) Klafter, J.; Silbey, R. J. Chem. Phys. 1980, 72, 849.

(26) Avramov, I.; Milchev, A.; Argyrakis, P. Phys. Rev. E 1993, 47, 2303

(27) Sigal, H.; Markovitsi, D. Unpublished results.

JP9600773 